\title{
Samples of stimuli, responses, and reinforcers: Effect of incongruent sample type, serial position, and mode of presentation
}

\author{
DOUGLAS S. GRANT \\ University of Alberta, Edmonton, Alberta T6G 2E9, Canada
}

\begin{abstract}
In two matching-to-sample experiments, pigeons' performance with samples of stimuli (red and green), number of responses ( 1 and 20 ), and reinforcers (food and no food) was assessed. Samples of red, 20 responses, and food were associated with the red comparison stimulus, and samples of green, 1 response, and no food were associated with the green comparison stimulus. On interference trials, three sample types were presented on each trial, and two of the samples (congruent) were associated with the correct comparison and the third sample (incongruent), with the incorrect comparison. Performance on interference trials was compared with that on control trials in which either two (Experiment 1) or three (Experiment 2) congruent samples were presented. It was found that presentation of an incongruent sample reduced matching accuracy markedly, and about equally, whether samples were presented successively or in compound. Although the type of sample that was incongruent was without effect, matching accuracy declined strongly as the recency of the incongruent sample increased. Serial position of the incongruent sample also influenced the shape of the retention function on interference trials. Presentation of the incongruent sample either first or second resulted in accuracy decreasing across the retention interval, whereas presentation of the incongruent sample last in the input sequence resulted in increasing accuracy across the retention interval. The theoretical implications of the findings are considered.
\end{abstract}

Several studies have employed a variant of the delayed matching-to-sample procedure, called the intratrial preparation, to investigate proactive interference in the pigeon. Performance on interference trials, in which two different samples are presented successively, is compared with that on control trials, in which only a single sample is presented. On interference trials, a single response to the comparison stimulus that matches the second, more recent sample defines a correct response. An interference effect is demonstrated to the extent that matching accuracy is lower on interference trials than on control trials.

Studies employing colored fields as sample and comparison stimuli have obtained a robust interference effect in the intratrial preparation only if the incorrect comparison corresponded to the sample presented initially on interference trials (Grant \& Roberts, 1973; Roberts \& Grant, 1974, Experiment 3; Zentall \& Hogan, 1974, 1977). This finding suggests that interference is not a function of the initial sample's either preventing storage of information derived from the second sample or degrading that information once storage is complete. Instead, interfer-

This work was supported by Grant A0443 from the Natural Sciences and Engineering Research Council of Canada. The technical assistance of Linda Scharr is gratefully acknowledged. Requests for reprints should be sent to: Douglas S. Grant, Department of Psychology, University of Alberta, Edmonton, Alberta T6G 2E9, Canada. ence may be attributed to a process of competition in which information derived from the initial sample controls choice responding on some proportion of trials (Grant \& Roberts, 1973; Roberts \& Grant, 1976). In the absence of a match between the incorrect comparison and the initial sample, there would be no opportunity for control by the initial sample and hence no reduction in matching accuracy relative to that on control trials.

Grant (Note 1) has demonstrated recently that the occurrence of intratrial interference is not restricted to the case in which colored fields are employed as stimuli. In his initial experiment, line orientation and color stimuli were each employed exclusively on some trials and were employed within the same trial on other trials. In a second experiment, samples of number of responses and of food and no food (see Maki, Moe, \& Bierley, 1977) were employed. As in the first experiment, each sample type was employed exclusively on some trials and sample types were mixed on other trials. Accuracy in each of the three interference trial types in both experiments was reduced relative to that in the appropriate control trial type.

The present experiments extended the preparation employed by Grant (Note 1) to investigate intratrial interference further. As a function of prior training, the birds reliably selected the red comparison when given a sample of a red field, 20 responses to a white circle, or the occurrence of food, and reliably 
selected the green comparison when given a sample of a green field, one response to a white circle, or the occurrence of no food. In the conditions of primary interest, three nominally different samples were presented on each interference trial, two of which were associated with the correct comparison and the other, with the incorrect comparison. Thus, one of the three samples presented on interference trials was incongruent with the other two.

The presentation of three kinds of samples, any one of which could be associated with the incorrect comparison stimulus, permits additional questions concerning pigeon memory to be addressed. In the first experiment, the amount of control over choice responding exerted by the incongruent sample was assessed as a function of retention interval and the rapidity with which the samples were presented within a trial. The issue of whether interference is sensitive to either the type (color, number of responses, and food/no food) or the serial position (first, second, and third) of the incongruent sample was of primary concern in the second experiment.

\section{EXPERIMENT 1}

Birds were tested on two primary types of trials, control trials and interference trials. Three sample types were presented in the sequence color, number of responses, and food/no food on each interference trial. Two of the three samples were associated with the correct comparison stimulus (congruent samples), and the remaining sample was associated with the incorrect comparison stimulus (incongruent sample). The type of sample that was incongruent was varied over trials and was equally often color, number of responses, and food/no food. Because sample types were always presented in the same sequence, type and serial position of the incongruent sample were confounded. That is, an incongruent color sample was necessarily presented first in the sequence, an incongruent number of responses sample second, and an incongruent food/no-food sample third.

Matching accuracy on the three types of interference trials was assessed relative to that on three comparable types of control trials. Control trials were identical to interference trials except that only the two congruent sample types were presented. Thus, control trials involved the presentation of a color sample and a number of responses sample, a number of responses sample and a food/no-food sample, or a color sample and a food/no-food sample.

Two additional independent variables, mode of sample presentation and retention interval, were crossed factorially with the control-interference variable and with each other. Mode of sample presentation refers to the manner in which the sample types were presented (see Grant, in press). In the successive mode, each sample presentation was a discrete event and was preceded by a preparatory stimulus requiring a single response. In the compound mode, the sample types were presented in the most spatially and temporally contiguous arrangement possible, although absolute simultaneity could not be achieved. Retention intervals of $0,7.5,15$, and $30 \mathrm{sec}$ separated termination of the final sample in the sequence and presentation of the comparison stimuli.

The primary concern was whether the incongruent sample type would control choice responding when presented in the context of two congruent sample types and, if so, whether amount of control would be sensitive to sample presentation mode. It is reasonable to suggest that presentation of two congruent sample types might serve to mask or overshadow the presentation of a single incongruent sample type. Moreover, because the successive mode affords a longer period of time in which to extract information during input than does the compound mode, it was anticipated that any such masking effect might be enhanced on compound trials. If so, control by the incongruent sample type would be greater on interference trials involving the successive sample presentation mode.

The effect of incongruent sample type and serial position could not be assessed independently in the present experiment due to the confounding. However, a preliminary indication of whether either of these variables influenced performance was provided by the present design. The confound between type and serial position of the incongruent sample was broken in the second experiment, and the effect of each variable was assessed systematically.

\section{Method}

Subjects. Five Silver King pigeons were maintained at $80 \%$ of their free-feeding weights. All birds had had extensive prior experience in matching to samples of color, number of responses, and food/no food.

Apparatus. The birds were tested in a modular test chamber. Three pecking keys were mounted horizontally in a row $20 \mathrm{~cm}$ above the floor. An IEE in-line projector was mounted behind each key and was used to project stimuli onto the pecking key. A grain feeder was mounted below the center pecking key. The test chamber was enclosed in a sound- and light-attenuating enclosure. Masking noise was provided by an exhaust fan within the enclosure and by white noise delivered through a speaker in the testing room.

The presentation of events within the chamber was controlled by a photoelectric paper tape reader interfaced to solid-state control modules. Data were recorded on each trial by an electronic printing counter. The control equipment was located in a room adjacent to the testing room.

Procedure. Because the birds had had extensive prior experience matching to samples of stimuli, responses, and reinforcers, little preliminary training was required. The birds received eight sessions of training prior to the experiment; the four odd-numbered sessions involved the successive sample presentation mode, and the four even-numbered sessions involved the compound sample presentation mode. In the successive mode, trials began with the illumination of the center key with the preparatory dot stimulus. A single response to the center key replaced the dot with a color sample that remained for a fixed duration of $2 \mathrm{sec}$. The preparatory 
dot was again presented immediately after the color sample, and a single response replaced the dot with the white circle. After either 1 or 20 responses to the circle, the dot appeared once more and a single response produced a 2 -sec food or no-food sample. On compound trials, the dot was presented only at trial onset. Following a response, the color sample appeared and remained illuminated until either 1 or 20 responses had occurred. The final response to the color sample terminated the color sample and produced immediately a 2-sec period of food or no food.

During preliminary training, the sample sequence was either red, 20 responses, and food or green, 1 response, and no food. As a function of prior training, samples of red, 20 responses, and food were each associated with the red comparison stimulus. On the other hand, samples of green, 1 response, and no food were each associated with the green comparison stimulus. During preliminary training, then, all three samples presented on each trial were consistent with the same comparison response. Following presentation of the final sample on both successive and compound trials, a retention interval of $0,3,6$, or $12 \mathrm{sec}$ preceded presentation of a red field and a green field on the side keys for a choice response. A single response to the red comparison produced 2 -sec access to grain following presentation of the red/20 responses/food sample sequence, and a single response to the green comparison produced 2-sec access to grain following presentation of the green/1 response/ no-food sample sequence. A single response to the incorrect comparison produced a $2-\mathrm{sec}$ time-out. An intertrial interval of $30 \mathrm{sec}$ separated trials within a session.

Two main types of trials were employed in the experiment, control and interference. Interference trials were identical to those of preliminary training except that one of the three sample types was associated with the alternate comparison stimulus. On such trials, the correct comparison stimulus was that which was associated with two of the three sample types. The type/serial position of the incongruent sample was equally often color first, number of responses second, and food/no food third. Three types of corresponding control trials involved the presentation of the two congruent sample types only. The sample type not presented on control trials was equally often color, number of responses, and food/ no food. Trials on which a color sample was not presented involved presentation of the white circle as the stimulus to which keypecks during the number of responses sample were directed.

Retention was tested at intervals of $0,7.5,15$, and $30 \mathrm{sec}$, and the intertrial interval was $45 \mathrm{sec}$. A $28-\mathrm{V}$ houselight, mounted on the intelligence panel, provided dim ambient illumination throughout each session except during the presentation of food/no-food samples and during periods of reinforcement and time-out.

A total of 96 different trials were employed ( 2 conditions, control and interference, $\times 3$ trial types $\times 2$ correct comparison stimuli $\times 2$ positions of the correct comparison stimulus $\times 4$ retention intervals). Eight sequences of 60 trials were prepared; four involved successive sample presentations, and four involved compound sample presentations. The first 12 trials of each sequence were identical to those of preliminary training, and data were not recorded on these trials. Of the remaining 48 trials, 24 were control (two from each of the three trial types at each retention interval) and 24 were interference (again, two from each of the three trial types at each retention interval). Each bird was tested on each of the trial sequences once every eight sessions, and sessions alternated between the two sample presentation modes. Across the 24-session experiment, each bird was tested at each retention interval within each of the three control and three interference trial types 48 times, 24 times within each mode of sample presentation.

\section{Results}

Percentage of correct responses as a function of retention interval are shown in Figure 1 for each of the three trial types within each condition. Data obtained on successive presentation mode trials are shown in the left panel, and those obtained on compound presentation mode trials are shown in the right panel. The three types of control trials are labeled according to which of the three sample types was not presented on that trial. The three interference trial types are labeled according to the type and serial position of the incongruent sample.

A conditions $\times$ type $\times$ retention interval $\times$ mode of sample presentation analysis of variance revealed that performance was more accurate on control trials than on interference trials $[F(1,4)=74.30, p<.001]$ but was unaffected by the mode of sample presentation $(F<1)$. Matching accuracy was influenced by the type of sample that was omitted on control trials and by the type of sample that was incongruent on interference trials $[F(2,8)=18.47, p<.001]$. Specifically, matching was least accurate within conditions when the omitted sample or incongruent sample was food/ no food. However, performance on interference trials was more strongly affected by the type factor than was performance on control trials [conditions $\times$ type interaction, $F(2,8)=16.28, p<.01]$.

Although accuracy generally declined as a function of retention interval $[F(3,12)=59.05, p<.001]$, the rate of decline was greater on control trials [conditions $x$ retention interval interaction, $F(3,12)=14.92$, $\mathrm{p}<.001$ ]. In fact, accuracy actually increased as retention interval lengthened when the food/no-food sample type was incongruent. The latter finding resulted in a significant triple interaction involving the factors of condition, type, and retention interval $[F(6,24)=11.07, p<.001]$.

The data were analyzed further by performing three separate analyses in which each interference trial type was compared individually with the appropriate control trial type. Accuracy was significantly lower when any of the three sample types was incongruent than when that sample type was omitted $[\mathrm{Fs}(1,4)=30.18$, $14.67,28.86$, ps $<.01, .05$, and .001 , for color, number

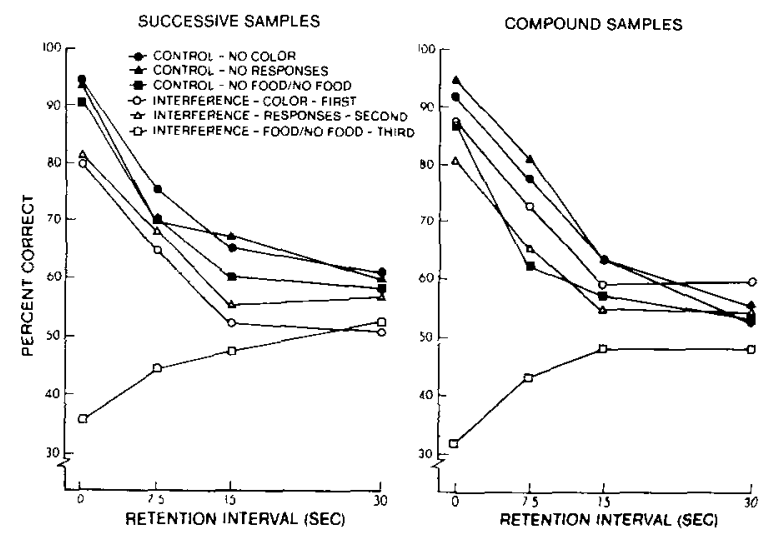

Figure 1. Percentage of correct responses in Experiment 1 as a function of retention interval with type of trial as the parameter. Data from trials employing the successive sample presentation mode are shown in the left panel, and data from trials employing the compound sample presentation mode are shown in the right panel. 
of responses, and food/no-food sample types, respectively]. The analyses revealed further that the relationship between control and interference accuracy changed across the retention interval only when the food/no-food sample type was involved $[F(3,12)=$ $36.63, \mathrm{p}<.001]$. Specifically, as retention interval was lengthened, accuracy decreased monotonically when the food/no-food sample type was omitted but increased monotonically when the food/no-food sample type was incongruent.

\section{Discussion}

The main finding from the present experiment was that matching was less accurate when any of the three sample types was incongruent than when that sample type was omitted. In fact, when the final sample type in the sequence (food/no food) was incongruent and the retention interval was short, the birds selected the comparison stimulus associated with that sample on a clear majority of trials. These findings suggest that the incongruent sample type does exert control over choice responding on some proportion of trials in the present preparation. The failure of mode of sample presentation to influence performance suggests further that the degree of control exerted by the incongruent sample is independent of the rate at which sample types are presented within a trial.

The amount of control exerted by the incongruent sample was strongly influenced both by length of retention interval and by the type/serial position of the incongruent sample. Particularly intriguing was the finding that accuracy increased as retention interval increased when the final sample in the sequence was incongruent. Because type and serial position were confounded, it cannot be determined which of these factors was responsible for the effect. Until such a determination has been made, theoretical speculation concerning the decreasing control by the incongruent sample over time would be premature. The second experiment investigated independently the effect of type and serial position.

\section{EXPERIMENT 2}

The confounding present in the preceding experiment between type and serial position of the incongruent sample was necessitated by the desire to present the sample types in the most spatially and temporally contiguous arrangement possible on compound trials. The ideal sample sequence from this point of view is, of course, color, number of responses, and food/no food. The purpose of the present experiment was to assess independently the effect of type and serial position of the incongruent sample. This was accomplished by breaking the confound and presenting each type of incongruent sample equally often at each of the three serial positions. This proce- dural change required that samples be presented successively on all trials.

There is reasonable justification for anticipating that both the type and the serial position of the incongruent sample will influence matching performance. Consider first the type of incongruent sample, which could be color, number of responses, or food/no food. Grant (in press), using the same sample of subjects as employed in the present experiments, assessed matching accuracy on trials on which only a single sample type was presented. Relative to samples of color and number of responses, samples of food and no food controlled higher levels of matching accuracy. This finding suggests that interference might be particularly robust when the incongruent sample is of the food/ no-food type.

Recent studies of memory for lists of stimuli in animals suggest that serial position plays a major role in determining performance. In the pigeon, Shimp and Moffitt $(1974,1977)$, employing a probe recognition procedure, have demonstrated a marked recency effect. That is, retention was best for the item presented last in a three-item list and was progressively less for the second and first items. A similar recency effect has been obtained by Thompson and Herman (1977), who presented six-item lists of auditory stimuli to a dolphin. Recent studies employing monkeys have demonstrated primacy as well as recency effects (Sands \& Wright, 1980; Roberts \& Kraemer, 1981). That is, both the initial and terminal list items were retained better than were items presented in the middle of the list. Finally, Wagner and Pfautz (1978), employing New Zealand rabbits, found that habituation occurred more slowly to stimuli presented at the middle positions of a serial list than to stimuli presented at either the initial or terminal serial positions. Thus, both primacy and recency effects have been demonstrated in primate and nonprimate species, although only recency effects have been demonstrated in avian species.

These findings suggest that the serial position of the incongruent sample will also influence the amount of interference produced in the present experiment. To date, only recency effects have been demonstrated in the pigeon, and, therefore, it was anticipated that interference would be maximal when the incongruent sample was presented third in the input sequence. In addition, there was particular interest in whether or not a primacy effect would be obtained. To the extent that the present preparation is more sensitive to primacy effects than is the preparation employed by Shimp and Moffitt (1974, 1977), matching accuracy might be lower when the initial sample is incongruent than when the second sample is incongruent.

\section{Method}

The same subjects and apparatus were again employed. Interference trials involved 36 different sample sequences created by the 
factorial combination of three incongruent sample serial positions, three incongruent sample types, two orders in which the congruent sample types could be presented, and two levels of correct comparison sequences (red correct sequences and green correct sequences). Control trials involved 12 different sample sequences created by presenting the three sample types in all possible orders within both red correct and green correct sample sequences. Combined factorially with the sample sequence factor was retention interval $(0,3,6$, and $12 \mathrm{sec})$ and position of the correct comparison stimulus. Therefore, a total of 288 different interference trials and 96 different control trials were employed. All trials involved the successive sample presentation mode.

Nine different sequences of 64 trials each were prepared. Each sequence contained 32 interference trials and 32 control trials, 8 at each retention interval within each type of trial. Across the nine sequences, each bird was tested on each interference trial once and on each control trial three times. Each bird was tested on each sequence every nine sessions and six times in the course of the 54-session experiment. Each bird was therefore tested 48 times at each retention interval within each of the nine combinations of type and serial position of the incongruent sample. All other aspects of the procedure were the same as in Experiment 1.

\section{Results}

Percentage of correct responses as a function of retention interval are shown in Figure 2. Performance on interference trials is shown as a function of incongruent sample serial position (collapsed across type) in the left panel and as a function of incongruent sample type (collapsed across serial position) in the right panel. Accuracy on control trials is plotted in each panel to facilitate comparison.

A type $\times$ serial position $\times$ retention interval analysis of variance was performed on the data from interference trials. The analysis revealed significant main effects of serial position $[F(2,8)=35.05, \mathrm{p}<.001]$ and retention interval $[\mathrm{F}(3,12)=22.94, \mathrm{p}<.001]$, but the effect of type failed to approach significance $(F<1)$. Newman-Keuls tests $(\alpha=.05)$ were performed to assess differences in accuracy as a function of incongruent sample serial position. An incongruent sample presentation produced significantly greater interference when it was third in the sequence rather
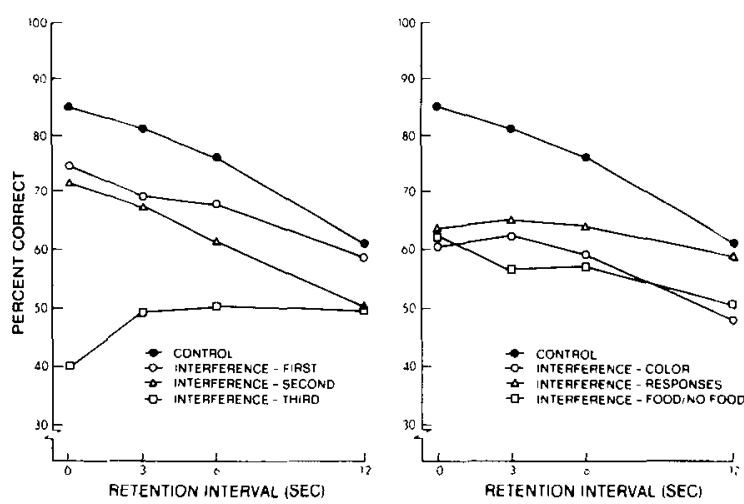

Figure 2. Percentage of correct responses in Experiment 2 as a function of retention interval. Performance on interference trials is shown as a function of incongruent sample serial position in the left panel and as a function of incongruent sample type in the right panel. than second and produced significantly greater interference when it was second in the sequence rather than first. The main analysis also revealed a significant serial position $\times$ retention interval interaction $[F(6,24)=7.67, p<.001]$. This interaction reflects primarily the finding that matching accuracy decreased monotonically across the retention interval when the incongruent sample was presented either first or second in the sequence. On the other hand, when the most recently presented sample was incongruent, matching accuracy was lowest on the immediate test and tended to increase as retention interval was lengthened.

The data were analyzed further by comparing each of the three serial position functions with the control. Each of the three analyses revealed that performance was more accurate on control trials than on interference trials $[F s(1,4)=18.45,59.15$, and 65.31 , ps $<.05, .01$, and .01, for first, second, and third serial positions, respectively] and that accuracy was influenced by retention interval $[\mathrm{Fs}(3,12)=96.42$, 40.08 , and 19.15 , ps $<.001$ in all cases]. In addition, the control-interference factor interacted significantly with retention interval when control and interference/third serial position accuracy was compared $[F(3,12)=20.48, p<.001]$.

\section{Discussion}

The primary concern of the present experiment was the effect of type and serial position of the incongruent sample. Although Grant (in press) has shown that samples of food and no food control higher levels of matching accuracy than are controlled by either color or number of responses samples, the amount of interference obtained in the present preparation was not affected by the type of incongruent sample. It is not immediately apparent as to how these two seemingly contradictory findings may be reconciled.

In contrast with type, the serial position of the incongruent sample influenced markedly the amount of control over choice responding exerted by that sample. One manifestation of the effect of serial position was revealed in terms of overall differences in matching accuracy. Specifically, matching accuracy decreased monotonically as the recency of the incongruent sample increased and was particularly low when the final sample in the sequence was incongruent. This finding is consistent with prior research in the pigeon (Shimp \& Moffitt, 1974, 1977) in demonstrating an effect of recency but no effect of primacy. Whether the primacy effect represents a phenomenon not produced by the pigeon information processing system or whether other preparations might reveal evidence for such an effect remains an open question.

In addition to influencing overall level of accuracy, incongruent sample serial position also affected the shape of the retention function. Accuracy decreased 
monotonically as retention interval increased when the incongruent sample was presented either first or second in the input sequence. In contrast, accuracy increased monotonically across the retention interval when the final sample in the sequence was incongruent. This trend was particularly apparent across the first $3 \mathrm{sec}$ of the retention interval.

\section{GENERAL DISCUSSION}

The initial presentation of a sample stimulus associated with the incorrect comparison stimulus has been found to reduce matching accuracy markedly (Grant \& Roberts, 1973; Zentall \& Hogan, 1974, 1977). The present experiments extend the generality of this interference phenomenon in a number of respects. First, the presentation of a sample associated with the incorrect comparison reduces matching accuracy even in the case in which two congruent samples, each associated with the correct comparison, are presented. Second, an incongruent sample controls choice responding, and thereby produces interference, to an equivalent degree regardless of the rapidity with which those samples are presented. Third, an incongruent sample reduces matching accuracy not only when that sample is presented first in the sequence, but also when it either is interpolated between two congruent samples or is presented following two congruent samples. Finally, the amount of control exerted by an incongruent sample increases as the serial position of that sample increases. In other words, an incongruent sample results in greater interference when presented later rather than earlier in the sample sequence.

None of these findings are likely to prove problematic for contemporary conceptions of animal short-term memory. For example, theories emphasizing processes of either temporal discrimination (D'Amato, 1973), trace strength and competition (Roberts \& Grant, 1976), or rehearsal (Grant, 1982; Wagner, 1978) account readily for the findings that (1) presentation of an incongruent sample reduces accuracy and (2) the magnitude of that reduction increases as the recency of incongruent sample presentation increases.

Likely to prove more theoretically intractable is the finding that incongruent sample serial position influenced the shape of the retention function. Presentation of an incongruent sample either first or second in the sample sequence resulted in statistically equivalent levels of interference across the retention interval. In contrast, an incongruent sample presented in the final input serial position resulted in maximal interference on an immediate test and progressively less interference as retention interval was lengthened.

Temporal discrimination and trace theory can account for decreasing interference across the retention interval when the incongruent sample is presented last, but both incorrectly predict the opposite effect when the incongruent sample is presented first. According to D'Amato's (1973) temporal discrimination view, animals discriminate at the time of testing which of several memories was established most recently. The memory that is discriminated as having been established most recently determines the choice response. The proportion of trials on which the comparison corresponding to the most recently established memory is selected should be greater at shorter than at longer retention intervals. This is the case because the ratio of time since establishment of the most recent and earlier memories is relatively greater at shorter intervals than at longer ones, resulting in more accurate temporal discrimination at shorter retention intervals.

Application of this view to the present preparation leads to the expectation that the sample presented last in the sequence should exert maximum control on the immediate test and progressively less control as retention interval is lengthened. If the incongruent sample is presented last, the incongruent memory should be discriminated as the most recent with a high probability on an immediate test, leading to control by that memory on a substantial proportion of trials. As the time since presentation of the sample sequences increases, it becomes increasingly more difficult to discriminate whether the congruent or incongruent memory was established most recently. Thus, as retention interval increases, the probability that the congruent memory will be incorrectly discriminated as having been established most recently will increase, leading to decreased control by the incongruent memory.

Consistent with the present findings, then, temporal discrimination theory predicts that presentation of an incongruent sample third in the sequence should result in decreasing interference across the retention interval. By the same reasoning, however, this view incorrectly anticipates that interference should be minimal on an immediate test and should increase progressively as retention interval is lengthened when the incongruent sample is presented first.

Consider next trace-strength theory and its application to the interference preparation (Grant, 1975; Grant \& Roberts, 1973; Roberts \& Grant, 1976). According to this view, presentation of a sample stimulus establishes a memory trace with a strength determined by the duration of sample presentation. The trace is held to lose strength as a negatively accelerated function of time in the absence of the sample. If traces associated with each of the comparison stimuli are present at testing, the stronger trace controls choice responding. However, because the strength of the trace established by an identical sample presentation is held to vary from occasion to occasion, the generally weaker of two traces may nevertheless control choice responding on some proportion of trials. Thus, 
the extent to which the sample associated with the incorrect comparison controls choice responding is related directly to the degree of overlap between the trace-strength distribution of that sample and that of the sample associated with the correct comparison.

If it is assumed that presenting the incongruent sample in the final serial position afforded an opportunity for decay of the congruent trace(s) prior to onset of decay of the incongruent trace, the data for the third serial position may be interpreted within the trace model. That is, the congruent memory would have lost considerable strength, and the incongruent memory little, at the 0 -sec retention interval. Because trace-strength loss follows a negatively accelerated course, the congruent memory would then lose strength less rapidly than the incongruent memory as the retention interval was lengthened. Thus, decreasing control by the incongruent sample as a function of retention interval would be anticipated. Once again, however, the same reasoning incorrectly predicts the reverse effect when the incongruent sample is presented in the initial serial position. In this case, the incongruent memory should lose strength less rapidly than the congruent memory and interference should increase across the retention interval. The strength model also anticipates that these effects would be markedly stronger when samples are presented successively, a notion without statistical support from the data of Experiment 1.

Although rehearsal models (Grant, 1982; Wagner, 1978) would likely not have led a priori to the expectation of a serial position by retention interval interaction, such models may afford the most adequate framework in which to interpret this finding. Grant (1982) has outlined a conception of pigeon shortterm memory in which sample stimuli are viewed as activating instructional memories (e.g., "peck red," "peck green"). Memories are held to return to the inactive state as a function of time following activation, but the period of activation may be prolonged by rehearsal or shortened by processing other incoming information. Choice behavior is viewed as controlled by memories active at testing, either those that have remained active throughout the retention interval or have returned to the active state via mechanisms of retrieval at testing.

In order to account for the present findings within this scheme, it is necessary to postulate that the birds entered the present experiments with a tendency to rehearse an instructional memory activated by more than one sample stimulus. This is a reasonable assumption in that the present subjects had experienced several months of sessions in which multiple congruent samples were presented on the majority of trials. During these sessions, then, processing of a multiply activated memory would have led to an increase in the probability of reinforcement at testing.
Consider first how the model would interpret the findings obtained when the incongruent sample was presented either first or second. The occurrence of a subsequent congruent sample or samples would result in the incongruent memory's being in an inactive state at the time of immediate test on the majority of trials, resulting in relatively low levels of interference. On the minority of trials in which both the congruent and incongruent memories were still active following completion of the sample sequence, differential rehearsal of the multiply activated congruent memory would tend to reduce the probability of control by the incongruent memory as time passed. However, because the incongruent memory is only rarely active following presentation of the complete sample sequence, differential rehearsal would influence performance only rarely. Thus, only a weak tendency toward decreasing interference with increasing retention interval would be anticipated.

Although not supported statistically, such a trend was present in all cases in the present experiments. In Experiment 1, control accuracy decreased by $31.8 \%$ and $34.8 \%$ across the retention interval on successive and compound trials, respectively. On interference/color-first trials, the decrease was only $29.3 \%$ and $27.5 \%$ and was $24.5 \%$ and $25.5 \%$ on interference/ responses-second trials. In Experiment 2, control accuracy decreased by $23.7 \%$ and by only $16.1 \%$ and $21.8 \%$ on interference/first and interference/second trials, respectively.

Presentation of the incongruent sample in the final serial position would be expected to produce marked interference on an immediate test because of the relatively high probability that the incongruent memory would be active and the congruent memory inactive. As in the case of an incongruent sample presented either first or second, a tendency to rehearse the multiply activated congruent memory on trials in which both memories were active would produce a weak trend toward decreasing interference with increasing retention interval. However, the trend would be enhanced markedly on interference/third trials if either the birds failed to rehearse the incongruent memory even when it was the only active memory or the congruent memory tended to remain active on a relatively large number of trials in spite of the subsequent presentation of the incongruent sample. In the former case, the incongruent memory would return to the inactive state rather quickly and performance would be controlled by processes of retrieval. In the latter case, multiple activation of the congruent memory might increase its resistance to retroactive interference. If so, differential rehearsal would occur more frequently when the incongruent sample was presented last in the sequence. This, in turn, would amplify the trend toward decreasing interference at longer retention intervals. 
Although speculative, the present interpretation does make testable predictions. For example, it should be possible to produce an increase in interference across the retention interval by presenting more incongruent sample types than congruent sample types within a trial. As a second example, the present conception suggests that conditions of initial training may influence the nature of the serial position by retention interval interaction. Extensive exposure to a preparation in which the multiply activated memory was associated with the incorrect comparison might lead to a tendency to differentially rehearse a memory activated by only a single sample. If so, testing such birds on conditions similar to those employed here should produce dramatic and highly specific changes in outcome.

\section{REFERENCE NOTES}

1. Grant, D. S. Intratrial proactive interference in pigeon shortterm memory: Effect of stimulus dimension and dimensional change. Manuscript submitted for publication, July 1981.

\section{REFERENCES}

D'Aмaто, M. R. Delayed matching and short-term memory in monkeys. In G. H. Bower (Ed.), The psychology of learning and motivation: Advances in research and theory (Vol. 7). New York: Academic Press, 1973.

Grant, D. S. Proactive interference in pigeon short-term memory. Journal of Experimental Psychology: Animal Behavior Processes, 1975, 1, 207-220.

Grant, D. S. Short-term memory in the pigeon. In N. E. Spear \& R. R. Miller (Eds.), Information processing in animals: Memory mechanisms. Hillsdale, N.J: Erlbaum, 1982.

Grant, D. S. Prospective vs retrospective coding of samples of stimuli, responses, and reinforcers in delayed matching with pigeons. Learning and Motivation, in press.

Grant, D. S., \& Roberts, W. A. Trace interaction in pigeon short-term memory. Journal of Experimental Psychology, 1973, 101, 21-29.

Maki, W. S., Moe, J. C., \& Bierley, C. M. Short-term memory for stimuli, responses, and reinforcers. Journal of Experimental Psychology: Animal Behavior Processes, 1977, 3, 156-177.

Robents, W. A., \& Grant, D. S. Short-term memory in the pigeon with presentation time precisely controlled. Learning and Motivation, 1974, 5, 393-408.

Roberts, W. A., \& Grant, D. S. Studies of short-term memory in the pigeon using the delayed matching-to-sample procedure. In D. L. Medin, W. A. Roberts, \& R. T. Davis (Eds.), Processes of animal memory. Hilisdale, N.J: Erlbaum, 1976.

Roberts, W. A., \& Kraemer, P. J. Recognition memory for lists of visual stimuli in monkeys and humans. Animal Learning \& Behavior, 1981, 9, 587-594.

SANDS, S. F., \& Wright, A. A. Serial probe recognition performance by a rhesus monkey and a human with 10 - and 20 -item lists. Journal of Experimental Psychology: Animal Behavior Processes, 1980, 6, 386-396.

Sнimp, C. P., \& MofriT, M. Short-term memory in the pigeon: Stimulus-response associations. Journal of the Experimental Analysis of Behavior, 1974, 22, 507-512.

Shimp, C. P., \& Moffirt, M. Short-term memory in the pigeon: Delayed-pair-comparison procedures and some results. Journal of the Experimental Analysis of Behavior, 1977, 28, 13-25.

Thompson, R. K. R., \& Herman, L. M. Memory for lists of sounds by the bottle-nosed dolphin: Convergence of memory processes with humans? Science, 1977, 195, 501-503.

WAaner, A. R. Expectancies and the priming of STM. In S. H. Hulse, H. Fowler, \& W. K. Honig (Eds.), Cognitive processes in animal behavior. Hillsdale, N.J: Erlbaum, 1978.

Wagner, A. R., \& Pfautz, P. L. A bowed serial-position function in habituation of sequential stimuli. Animal Learning \& Behavior, $1978,6,395-400$.

Zentall, T. R., \& Hogan, D. E. Memory in the pigeon: Proactive inhibition in a delayed matching task. Bulletin of the Psychonomic Society, 1974, 4, 109-112.

Zentall, T. R., \& Hogan, D. E. Short-term proactive inhibition in the pigeon. Learning and Motivation, 1977, 8, 367-386.

(Manuscript received May 29, 1981; revision accepted for publication October 12, 1981.) 